\title{
Rizóbios e fungo micorrízico arbuscular inoculados em leucena com aplicação de biofertilizantes PK, mais enxofre e Acidithiobacillus
}

\author{
Rhizobia and arbuscular mycorrhizal fungi inoculation on \\ leucena with PK biofertilizer plus sulfur and Acidithiobacillus
}

Emmanuella Vila Nova da Silva ${ }^{*}$, Newton Pereira Stamford ${ }^{2}$, Maria do Carmo Silva Barreto ${ }^{3}$, Wagner da Silva Oliveira' ${ }^{1}$ Rosa Livia Carvalho de Moraes ${ }^{4}$, Márcia do Vale Barreto Figueiredo ${ }^{4,5,6}$

'Universidade Federal Rural de Pernambuco (UFRPE), Rua Dom Manoel de Medeiros, s/n, Dois Irmãos, CEP 52171-900, Recife, PE, Brasil 2Departamento de Agronomia, Universidade Federal Rural de Pernambuco (UFRPE), Recife, PE, Brasil ${ }^{3}$ Centro de Tecnologias Estratégicas do Nordeste (CETENE), Recife, PE, Brasil 4Laboratōiro de Biologia de Solo Instituto Agronômico de Pernambuco (IPA), Recife, PE, Brasil

${ }^{5}$ Secretaria de Agricultura e

Desenvolvimento Agrărio de Alagoas (SEAGRI), Maceiō, AL, Brasil 'Bolsista de Produtividade em

Pesquisa do CNPq - Nivel 2

*autor correspondente

凶manuvilanova@yahoo.com.br
RESUMO: Este trabalho teve como objetivo avaliar o efeito da inoculação de rizóbios e Glomus etunicatum em leucena com aplicação de biofertilizantes de rochas fosfatada e potássica (PK) mais enxofre com Acidithiobacillus, visando maior nodulação e desenvolvimento das plantas. Os trabalhos foram desenvolvidos no Instituto Agronômico de Pernambuco-IPA. Foram utilizadas quatro estirpes de rizóbios (BR112, BR814 - padrão, IPA209 e NFB494), aplicando $2 \mathrm{~mL}$ da cultura líquida $\left(10^{-8} \mathrm{ufc} \mathrm{mL}^{-1}\right)$, inoculados com $10 \mathrm{~g}$ propágulos de Glomus etunicatum, contendo 70 glomerosporos e $10 \mathrm{~g}$ biofertilizante PK, de acordo com o tratamento. A colheita foi efetuada aos 120 dias após a germinação. Os resultados mostraram que as plantas de leucena inoculadas com a estirpe BR 814 + G. etunicatum + biofertilizante PK não apresentaram diferença significativa pelo teste de Tukey $(\mathrm{p}<0,05)$ para as variáveis matéria seca da parte aérea e $\mathrm{N}$ total acumulado, porém a apresentaram em relação à altura de planta. As plantas de leucena inoculadas com a NFB 494 + G. etunicatum + biofertilizante PK obtiveram maiores médias na matéria seca de nódulos. Na matéria seca da raiz, as plantas que receberam o tratamento -R+NPK (ausência de inoculação de rizóbio acrescido de adubo mineral NPK) apresentaram-se superiores em relação aos demais tratamentos.

PALAVRAS-CHAVE: Simbiose, Leucaena leucocephala, Glomus etunicatum, nodulação, rochas fosfatada e potássica, fixação de $\mathrm{N}_{2}$.
ABSTRACT: The purpose of this research was to evaluate the effect of Glomus etunicatum and Rhizobia inoculation on leucena with phosphate and potassium rocks plus sulfur and Acidithiobacillus in order to promote enhanced nodulation and plant growth. The experiments were accomplished at the Agronomical Institute of Pernambuco, Brazil. Four rhizobia strains were used (BR112, BR814 - standard, IPA209 and NFB494) with $2 \mathrm{~mL}$ of rhizobia liquid culture $\left(10^{-8}\right.$ ufc. $\left.\mathrm{mL}^{-1}\right)$, inoculated with $10 \mathrm{~g}$ propagules of Glomus etunicatum containing 70 glomerospores and $10 \mathrm{~g}$ PK biofertilizer, according to the treatment. The harvest was performed 120 days after germination. Results showed that leucena plants inoculated with BR $814+$ G. etunicatum $+P K$ biofertilizer did not show significant difference in the Tukey test applied to the dry matter of shoot (DMS) and accumulated nitrogen (AcN) variables, but they showed difference in relation to plant height. Leucena plants inoculated with NFB $494+G$. etunicatum $+P$ K biofertilizer presented the highest average values in dry matter of nodules (DMN). Regarding dry matter of root (DMR), the plants that received treatment $-R+N P K$ (Rhizobium inoculation absence, but with mineral fertilizer $N P K)$ showed better results in relation to the other treatments.

KEYWORDS: Symbiosis, Leucaena leucocephal, Glomus etunicatu, nodulation, phosphate and potassium rocks, $\mathrm{N}_{2}$ fixation. 


\section{Introdução}

O estudo do uso de novos insumos com potencial agrícola, como, por exemplo, as rochas fosfatadas, é de grande interesse, quando se pensa em uma agricultura sustentável (STAMFORD et al., 2005), já que o uso de fertilizantes, que têm altos custos, é bastante agressivo aos solos (SANCHEZ, 2002).

As rochas fosfatadas podem ser usadas como matériasprimas nas indústrias de fabricação de fertilizantes fosfatados de alta solubilidade, como fontes de fósforo para aplicação direta na agricultura, ou podem ser utilizados para a fabricação de biofertilizantes, com adição de enxofre inoculado com bactérias oxidantes de enxofre elementar (STAMFORD et al., 2005).

Os solos, juntamente com seus organismos, participam de modo contundente para a manutenção da vida e para o equilíbrio da biosfera. Dentre os microrganismos do solo, os fungos micorrízicos arbusculares (FMA), que podem também apresentar efeito sinérgico com bactérias fixadoras de $\mathrm{N}_{2}$ (BFN) em simbioses tripartes (fungo-planta-bactéria) (JESUS; SCHIAVO; FARIA, 2005; CARVALHO; MOREIRA, 2010; MOREIRA et al., 2010), e o sistema simbiótico rizóbio-leguminosa (a partir de BFN) se destacam, por exercerem significativo desempenho para a funcionalidade e manutenção dos ecossistemas naturais, manejados e degradados (SOUZA et al., 2006).

Contudo, sabe-se que existem outros sistemas fixadores de $\mathrm{N}_{2}$, como os microrganismos de vida livre e os microrganismos associativos (SANTOS et al., 2008; REIS et al., 2010).

Dentre as várias espécies de leguminosas arbustivas e arbóreas, a leucena (Leucaena leucocephala (Lam.) R de Wit.) apresenta maior amplitude de utilização. De acordo com Lima Filho, Drumond e Macedo (1992), o interesse pelas espécies leguminosas arbóreas tem aumentado principalmente pela capacidade de fixar $\mathrm{N}_{2}$. Suas folhas são forrageiras para o gado e a madeira pode ser utilizada como lenha (LORENZI et al., 2003).

A leucena também apresenta considerável resistência à salinidade e à estiagem, sendo uma das mais promissoras, principalmente, pelo seu potencial para usos, como: controle da erosão, produção de energia, recuperação de áreas degradadas, adubação verde e enriquecimento das camadas superiores do solo, em termos de matéria orgânica proveniente das folhas e vagens que caem naturalmente (STAMFORD; CARVALHO, 1999; DRUMOND; RIBASKI, 2010). É bastante utilizada, também, em sistemas agrossilviculturais (LIMA, 2005).

Nas associações com rizóbio e FMA, se estiverem trabalhando eficientemente, a demanda por nitrogênio será menor que na maioria das culturas (LIMA, 2005).

$\mathrm{O}$ presente trabalho teve como objetivo avaliar o efeito da inoculação de rizóbios e Glomus etunicatum (Becker \& Gerdeman) em leucena, com aplicação de biofertilizantes de rochas fosfatada e potássica (PK), mais enxofre com Acidithiobacillus, visando maior nodulação e desenvolvimento das plantas.

\section{Material e Métodos}

$\mathrm{O}$ experimento foi conduzido em casa de vegetação da sede do IPA, em vasos com o solo Neossolo Flúvico Eutrófico $\left(9 \mathrm{~kg} \mathrm{vaso}^{-1}\right)$, com $\mathrm{pH} 6,7$. O solo foi coletado a uma profundidade de $20 \mathrm{~cm}$, sendo proveniente da Estação Experimental de Serra Talhada ( $7^{\circ} 59^{\prime} 15^{\prime}$ ' S e $38^{\circ} 18^{\prime} 0$ " W GR e altitude de $431 \mathrm{~m}$ ). Foi seco ao ar e peneirado (5 mm), sendo submetido às análises física e química (Tabelas 1 e 2) no Laboratório de Fertilidade e Física de Solo do IPA. O solo utilizado não foi autoclavado, sendo assim mantida e preservada sua micro, meso e macrofauna.

O delineamento experimental foi em blocos casualizados, com arranjo fatorial $4 \times 2 \times 2$, com 4 repetições e 6 tratamentos adicionais, contendo 4 estirpes de rizóbios (Tabela 3), com e sem FMA (Glomus etunicatum) e com e sem biofertilizante de rocha fosfatada e potássica (PK), com enxofre inoculado com Acidithiobacillus sp. e os seguintes tratamentos adicionais: $(-\mathrm{R}-\mathrm{M}-\mathrm{B},-\mathrm{R}-\mathrm{M}+\mathrm{B},-\mathrm{R}+\mathrm{M}+\mathrm{B},-\mathrm{R}+\mathrm{M}-\mathrm{B},+\mathrm{R}+\mathrm{PK}$ e-R+NPK), totalizando 88 vasos. $\mathrm{O}$ sinal negativo (-) significa ausência de inoculação com rizóbios $(-\mathrm{R})$, fungo micorrízico (-M) ou o Biofertilizante PK (-B). O sinal de positivo (+) significa presença de inoculação; +NPK representa aplicação de adubo mineral misto (Nitrogênio, Fósforo e Potássio), calculado de acordo com Cavalcanti et al. (2008).

$\mathrm{Na}$ análise estatística, foi utilizado o Programa Sanest, realizando-se a análise de variância (ANOVA) e a comparação de médias com o teste de Tukey ( $\mathrm{p}>0,05)$.

A bactéria foi purificada no meio YMA (ágar, manitol e extrato de levedura), utilizando o indicador vermelho congo (VINCENT, 1970). Em seguida, a estirpe foi repicada para frascos Erlenmeyers de $250 \mathrm{~mL}$ (três frascos para cada estirpe) com meio YM (manitol - extrato de levedura), em agitador rotativo, com temperatura de $28{ }^{\circ} \mathrm{C}$ por um período de 3 dias, exceto para a estirpe NFB494, que ficou em agitação por 2 dias.

A quebra de dormência das sementes de leucena foi realizada em água a $80{ }^{\circ} \mathrm{C}$ por 5 segundos, de acordo com Teles et al. (2000), e, em seguida, a desinfestação foi efetuada conforme recomendação descrita por Hungria e Araújo (1994).

O plantio foi efetuado, utilizando-se 5 sementes vaso $^{-1}$, inoculadas com $2 \mathrm{~mL}$ da cultura líquida de rizóbios $\left(10^{-8}\right.$ ufc.mL $\left.L^{-1}\right)$. Nos tratamentos com G. etunicatum (10 g propágulos contendo 70 glomerosporos + pedaços de raízes colonizadas). A aplicação do biofertilizante, com a bactéria oxidante do enxofre Acidithiobacillus, foi realizada com adição de $10 \mathrm{~g}$ vaso $^{-1}$ do biofertilizante (PK).

$\mathrm{O}$ solo foi irrigado e a umidade mantida em capacidade de pote. Depois da germinação, foi aplicada, semanalmente, a solução nutritiva $\left(2 \mathrm{~mL} \mathrm{~kg} \mathrm{solo}{ }^{-1}\right)$, sem N (HOAGLAND;

Tabela 1. Características químicas do Neossolo Flúvico Eutrófico proveniente da Estação Experimental do IPA, no município de Serra Talhada, PE.

\begin{tabular}{|c|c|c|c|c|c|c|}
\hline \multirow{2}{*}{ Área } & $\mathbf{P}$ & $\mathbf{C a}$ & Mg & $\mathbf{K}$ & Al & \multirow{2}{*}{$\frac{p H}{\mathrm{H}_{2} \mathrm{O} \quad 1: 2,5}$} \\
\hline & $\mathrm{mg} \mathrm{dm}^{-3}$ & \multicolumn{4}{|c|}{ cmolc dm dm $^{-3}$} & \\
\hline Serra Talhada - PE & $>40$ & 4,85 & 1,80 & 0,51 & 0 & 6,70 \\
\hline
\end{tabular}


Tabela 2. Características físicas do Neossolo Flúvico Eutrófico proveniente da Estação Experimental do IPA, no município de Serra Talhada, PE.

\begin{tabular}{|c|c|c|c|c|c|c|c|}
\hline \multirow{2}{*}{ Área } & \multicolumn{3}{|c|}{ Granulometria (\%) } & \multirow{2}{*}{ Classe textural } & \multirow{2}{*}{$\frac{\text { Umidade residual }}{\%}$} & Ds* & \multirow[t]{2}{*}{ Dp*** } \\
\hline & Areia & Argila & Silte & & & & \\
\hline Serra Talhada - PE & 79,20 & 12,80 & 8,00 & Franco arenoso & 0,94 & 1,55 & 2,70 \\
\hline
\end{tabular}

*Densidade do solo (ds), **Densidade da partícula (dp).

Tabela 3. Descrição da listagem das estirpes de rizóbios utilizadas na inoculação em Neossolo Flúvico Eutrófico.

\begin{tabular}{ll}
\hline $\begin{array}{c}\text { Estirpes } \\
\text { (Rizóbios) }\end{array}$ & \multicolumn{1}{c}{ Procedência } \\
\hline NFB 494 & $\begin{array}{l}\text { Núcleo de Fixação Biológica dos Trópicos da } \\
\text { Universidade Federal Rural de Pernambuco - UFRPE }\end{array}$ \\
BR 814 & Centro Nacional de Pesquisa em Agrobiologia - CNPAB \\
BR 112 & Centro Nacional de Pesquisa em Agrobiologia - CNPAB \\
IPA 209 & Instituto Agronômico de Pernambuco-IPA \\
\hline
\end{tabular}

ARNON, 1950). Foi efetuado o desbaste, deixando-se 1 planta vaso $^{-1}$.

A colheita foi efetuada aos 120 dias após a germinação (DAG), e a secagem do material vegetal de leucena foi efetuada em estufa a $60{ }^{\circ} \mathrm{C}$ por 72 horas. Foram avaliadas as seguintes variáveis: altura da planta (ALT), matéria seca da parte aérea (MSPA), matéria seca dos nódulos (MSN), matéria seca da raiz (MSR), relação da matéria seca da raiz pela matéria seca da parte aérea (MSR/MSPA), nitrogênio acumulado $\left(\mathrm{N}_{\mathrm{ac}}\right)$, teor de nitrogênio (TN) e eficiência da fixação de $\mathrm{N}_{2}\left(\mathrm{EFN}_{2}\right)$. Para análise do $\mathrm{N}$ total, o material vegetal foi moído (Moinho MR340, com malha 20) e, em seguida, calculado o $\mathrm{N}$ total acumulado na matéria seca da parte aérea, que foi determinado pelo método de Kjeldahl, segundo Bremner (1965).

\section{Resultados e Discussão}

$\mathrm{Na}$ Tabela 4, encontram-se os valores relacionados às variáveis (ALT); (MSPA); $\left(\mathrm{N}_{\mathrm{ac}}\right) ; \mathrm{e}\left(\mathrm{EFN}_{2}\right)$.

A produção de MSPA e $\mathrm{o} \mathrm{N}_{\mathrm{ac}}$ da leucena não apresentaram diferença estatística significativa $(p<0,05)$ entre os tratamentos que receberam inoculação de microrganismos e os que não receberam. Porém os tratamentos que receberam inoculação, como o BR814+M+B (rizóbio + FMA: Glomus etunicatum + biofertilizante PK), foram semelhantes aos tratamentos com rizóbio BR814 (padrão) e adubo mineral PK $(+\mathrm{R}+\mathrm{PK})$ e a adubação mineral NPK $(-\mathrm{R}+\mathrm{NPK})$ Moura et al. (2007). Para biomassa seca da parte aérea, em melão cultivado em Argissolo no Vale do São Francisco, encontraram-se resultados semelhantes, avaliando-se o efeito dos biofertilizantes de rochas fosfatadas e potássicas em comparação aos fertilizantes minerais (SFT e $\mathrm{KCl}$ ).

Andrade et al. (2009), ao aplicarem o tratamento mistura com fertilizante NPK mineral em caupi (Vigna unguiculata L. Walp), obtiveram menores proporções no acúmulo de biomassa seca da parte aérea, especialmente quando comparado com a aplicação de biofertilizante, mais a inoculação com Bradyrhizobium.

Resultados semelhantes foram obtidos por Stamford et al. (2004) trabalhando com caupi, nos quais se verificou que a produção de MSPA, utilizando-se superfosfato triplo e os biofertilizantes de rocha fosfatada (BP) com adição de enxofre, inoculados com rizóbio, apresentaram diferença significativa em relação aos tratamentos sem inoculação de rizóbio. Stamford et al. (2006), aplicando biofertilizantes com $\mathrm{P}$ e K (BP) e (BK), obtiveram resposta na produção de MSPA na cana-de-açúcar em pesquisa realizada em solo de tabuleiro da Zona da Mata de Pernambuco, inclusive com resultados superiores aos do tratamento com aplicação de fertilizantes minerais $(\mathrm{SFT}+\mathrm{KCL})$.

Considerados uma alternativa para a adubação, reforça-se, desta forma, a utilização desses microrganismos em conjunto com o Biofertilizante PK que podem, provavelmente, favorecer a microflora e microfauna do solo, tendo em vista que são produtos naturais com solubilização mais lenta de nutrientes. Por outro lado, pode ser considerado que, os biofertilizantes de rochas apresentem resultados similares aos dos fertilizantes convencionais, que, além de terem custo mais elevado, são bastante agressivos ao solo, em função de sua rápida solubilização e grande possibilidade de arraste de elementos químicos para o solo e para o lençol freático.

Jesus, Schiavo e Faria (2005), em experimento com a leguminosa Piptadenia gonoacantha, constatou que as plantas dos tratamentos BR3452 + FMA e BR4812 + FMA, inoculadas simultaneamente com rizóbios e FMAs, apresentaram maior produção de MSPA, em relação às plantas dos tratamentos BR3452 e BR4812, inoculadas apenas com rizóbio. Resposta semelhante, o mesmo autor obteve no experimento com a leguminosa, Piptadenia paniculata, em que as plantas dos tratamentos BR3405 + FMA e BR4829 + FMA, também apresentaram maior produção de MSPA.

Lima et al. (2007), trabalhando com MSPA de alface em solo do Cariri cearense, com biofertilizantes de rochas fosfatadas e potássicas, encontrou resultado semelhante aos obtidos no presente trabalho.

Silva et al. (2011), avaliando a disponibilidade de P para a cultura do melão em dois solos do Rio Grande do Norte, com o uso dos fertilizantes superfosfato triplo e biofertilizante de rocha fosfatada, observaram que não houve diferença significativa na produção de matéria seca, porém o biofertilizante foi o que mais disponibilizou $\mathrm{P}$ nos dois solos estudados.

Já em relação às variáveis altura de plantas e $\mathrm{EFN}_{2}$, as plantas de leucena inoculadas com a estirpe de rizóbio BR 814 - padrão, associadas ao Glomus etunicatum e ao biofertilizante PK (BR814+M+B), apresentaram-se superiores 
em relação aos demais tratamentos, embora os tratamentos com a estirpe de rizóbio BR 814, associada ao Glomus etunicatum (BR814+M-B) e o BR814, com ausência de inoculação com Glomus etunicatum e biofertilizante PK (BR814-M-B), não apresentassem diferença significativa $(\mathrm{p}<0,05)$, quando comparados com o BR814+M+B.

Os resultados sugerem a presença de FMA nativo do solo, tendo em vista que as amostras de solo não foram autoclavadas. Outra hipótese que deve ser considerada é a de que o solo usado apresenta uma quantidade relativamente adequada de fósforo disponível (>40 $\mathrm{mg} \mathrm{dm}^{-3}$ ) e pode ser um reservatório para a cultura, fazendo com que o P-lábil passe para a solução do solo (P-solução), agindo como um sistema tampão, não sendo favorável para atuação efetiva do fungo micorrízico.

$\mathrm{Na}$ Tabela 5, as plantas inoculadas com a estirpe de rizóbio (NFB 494) associada ao Glomus etunicatum e ao biofertilizante PK (Figura 1) apresentaram um dos melhores resultados quando comparados aos demais tratamentos, embora não tenha apresentado diferença significativa pelo teste de Tukey $(\mathrm{p}<$
0,05) para MSN. Obter maior nodulação foi um dos objetivos deste trabalho e, com este tratamento, foi possível confirmar a forte atuação destes microrganismos, favorecendo maior MSN, que reflete maior absorção do $\mathrm{N}_{2}$ pelas plantas, substituindo ao menos parcialmente, a utilização de fertilizantes minerais.

Andrade et al. (2009), trabalhando com caupi em um Argissolo da Zona da Mata de Pernambuco, avaliando o efeito da aplicação de fertilizante mineral e biofertilizante de rochas (BPK), na produção de biomassa seca de nódulos $(\mathrm{BSN})$, verificaram que a aplicação de $\mathrm{BPK}$ favoreceu à $\mathrm{BSN}$, enquanto a adição de fertilizante mineral promoveu inibição da nodulação.

Com relação ao teor de nitrogênio $(\mathrm{TN})$ na parte aérea de leucena, foi verificado que não houve diferença significativa $(\mathrm{p}<0,05)$ entre os tratamentos, apesar de se notarem os resultados superiores das plantas inoculadas com a estirpe de rizóbio (BR 112), associadas ao Glomus etunicatum, particularmente nos tratamentos com biofertilizante de rocha fosfatada e potássica, mais enxofre com Acidithiobacillus.

Tabela 4. Altura de planta (ALT), matéria seca da parte aérea (MSPA), eficiência de nitrogênio ( $\mathrm{EFN}_{2}$ ) e nitrogênio acumulado na parte aérea ( $\mathrm{N}_{\mathrm{ac}}$ ) em leucena com e sem inoculação, com estirpe de rizóbio (NFB494, BR 814 (padrão), BR 112 e IPA 209), Glomus etunicatum e Biofertilizante PK ${ }^{(1)}$.

\begin{tabular}{|c|c|c|c|c|}
\hline \multirow{2}{*}{ Tratamentos } & ALT & MSPA & $\mathrm{EFN}_{2}$ & \multirow{2}{*}{$\frac{\mathrm{N}_{\mathrm{ac}}}{\left(\mathrm{mg} \mathrm{N} \text { vaso }^{-1}\right)}$} \\
\hline & $(\mathbf{c m})$ & $\left(\right.$ g vaso $\left.^{-1}\right)$ & $\left(\mathbf{m g ~ N}\right.$ vaso $\left.^{-1} \mathrm{MSN}^{-1}\right)$ & \\
\hline NFB $494-M-B$ & $132,2 \mathrm{ab}$ & $18,4 \mathrm{a}$ & 674,2 def & 584,4 a \\
\hline NFB $494-M+B$ & $137,0 \mathrm{ab}$ & $17,9 \mathrm{a}$ & $593,5 \mathrm{f}$ & $567,2 \mathrm{a}$ \\
\hline NFB $494+\mathrm{M}+\mathrm{B}$ & $133,5 \mathrm{ab}$ & $17,8 \mathrm{a}$ & 616,9 ef & 590,7 a \\
\hline NFB $494+\mathrm{M}-\mathrm{B}$ & $126,7 \mathrm{ab}$ & $16,9 \mathrm{a}$ & 705,8 cdef & 548,5 a \\
\hline BR 814 -M-B & $145,2 \mathrm{a}$ & $20,7 \mathrm{a}$ & $1119,4 \mathrm{abc}$ & 649,3 a \\
\hline BR $814-M+B$ & $140,0 \mathrm{ab}$ & $19,5 \mathrm{a}$ & 1046,7 abcd & 602,7 a \\
\hline BR $814+\mathrm{M}+\mathrm{B}$ & $145,5 \mathrm{a}$ & $20,2 \mathrm{a}$ & $1172,5 \mathrm{a}$ & 639,1 a \\
\hline BR $814+\mathrm{M}-\mathrm{B}$ & $144,2 \mathrm{a}$ & $20,7 \mathrm{a}$ & $1127,2 \mathrm{abc}$ & $652,0 \mathrm{a}$ \\
\hline BR $112-M-B$ & $131,5 \mathrm{ab}$ & $16,8 \mathrm{a}$ & 617,7 ef & $543,1 \mathrm{a}$ \\
\hline BR $112-\mathrm{M}+\mathrm{B}$ & $132,0 \mathrm{ab}$ & $17,6 \mathrm{a}$ & 782,5 abcdef & 554,2 a \\
\hline BR $112+\mathrm{M}+\mathrm{B}$ & $134,2 \mathrm{ab}$ & $17,4 \mathrm{a}$ & 719,5 cdef & $577,0 \mathrm{a}$ \\
\hline BR $112+\mathrm{M}-\mathrm{B}$ & $129,7 \mathrm{ab}$ & $17,1 \mathrm{a}$ & 664,9 def & $562,5 \mathrm{a}$ \\
\hline IPA $209-\mathrm{M}-\mathrm{B}$ & $132,5 \mathrm{ab}$ & $18,4 \mathrm{a}$ & 737,2 cdef & 599,0 a \\
\hline IPA $209-\mathrm{M}+\mathrm{B}$ & $135,2 \mathrm{ab}$ & $18,9 \mathrm{a}$ & 743,3 bcdef & 604,6 a \\
\hline IPA $209+\mathrm{M}+\mathrm{B}$ & $131,2 \mathrm{ab}$ & $18,6 \mathrm{a}$ & 767,2 abcdef & $602,0 \mathrm{a}$ \\
\hline IPA $209+\mathrm{M}-\mathrm{B}$ & $133,5 \mathrm{ab}$ & $18,3 \mathrm{a}$ & $676,4 \mathrm{def}$ & 594,2 a \\
\hline$-\mathrm{R}-\mathrm{M}-\mathrm{B}$ & $122,7 \mathrm{~b}$ & $15,6 \mathrm{a}$ & $575,7 \mathrm{f}$ & 514,0 a \\
\hline$-\mathrm{R}-\mathrm{M}+\mathrm{B}$ & $139,5 \mathrm{ab}$ & $18,3 \mathrm{a}$ & $589,4 \mathrm{f}$ & 558,1 a \\
\hline$-\mathrm{R}+\mathrm{M}+\mathrm{B}$ & $131,5 \mathrm{ab}$ & 17,6 a & 678,2 def & $572,8 \mathrm{a}$ \\
\hline$-\mathrm{R}+\mathrm{M}-\mathrm{B}$ & $132,2 \mathrm{ab}$ & $16,0 \mathrm{a}$ & 758,9 abcdef & 509,1 a \\
\hline$+\mathrm{R}+\mathrm{PK}$ & $144,0 \mathrm{ab}$ & $21,3 \mathrm{a}$ & $1165,3 \mathrm{ab}$ & 645,4 a \\
\hline$-\mathrm{R}+\mathrm{NPK}$ & $138,7 \mathrm{ab}$ & $21,0 \mathrm{a}$ & 1024,9 abcde & $595,0 \mathrm{a}$ \\
\hline $\mathrm{CV}(\%)$ & 5,93 & 12,37 & 20,11 & 13,39 \\
\hline
\end{tabular}

${ }^{(1)} \mathrm{M}$ : fungo micorrízico arbuscular (Glomus etunicatum); B: biofertilizante de rocha fosfatada e potássica (PK), com enxofre inoculado com Acidithiobacillus; R: estirpe de rizóbio padrão; PK: adubo mineral - fósforo e potássio; NPK: adubo mineral - nitrogênio, fósforo e potássio; +: presente; -: ausente. Na coluna, as médias ( 3 repetições) seguidas da mesma letra não diferem estatisticamente entre si, ao nível de 5\% de probabilidade, pelo teste de Tukey. 

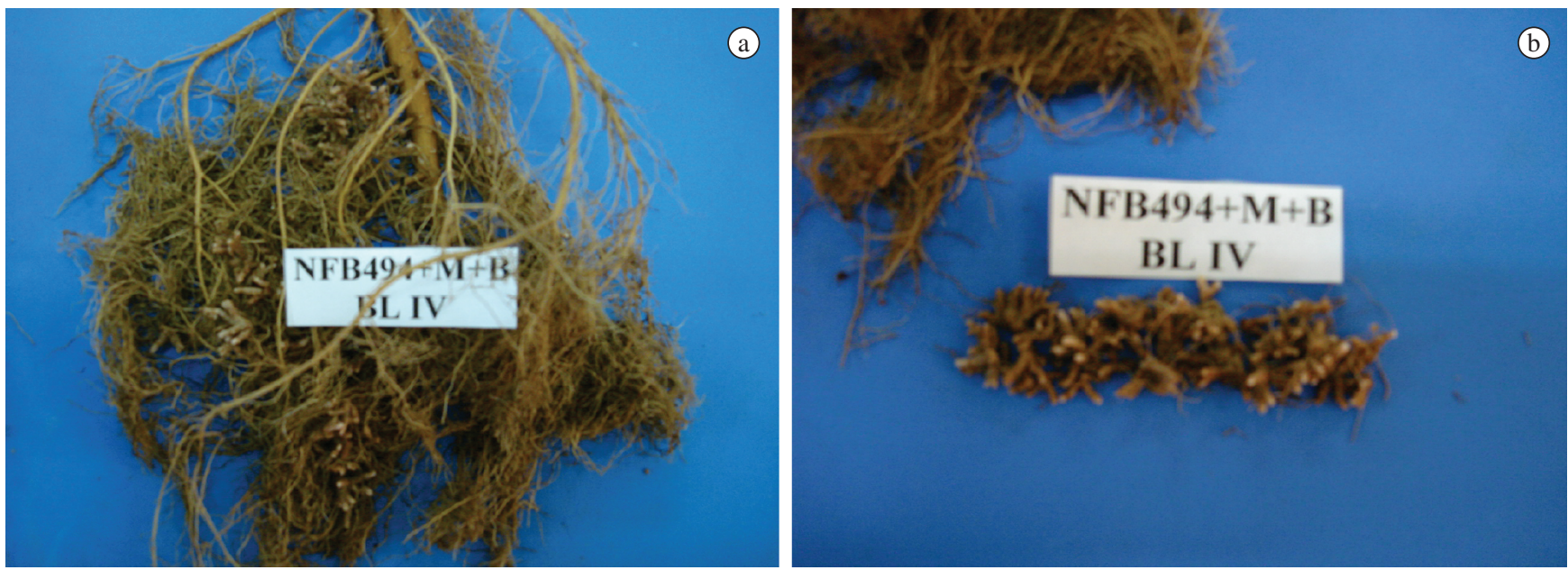

Figura 1. Sistema radicular de leucena (Leucaena leucocephala (Lam.) R de Wit.): a) Tratamento inoculado com NFB494+M+B, em que NFB 494 (rizóbio), $+\mathrm{M}$ = fungo micorrízico arbuscular (Glomus etunicatum) $+\mathrm{B}=$ biofertilizante de rocha fosfatada e potássica (PK); b) Com destaque para os nódulos e todos os tratamentos aos 120 dias após germinação (DAG).

Tabela 5. Matéria seca da raiz (MSR), matéria seca dos nódulos (MSN), teor de nitrogênio (TN), relação matéria seca da raiz pela matéria seca da parte aérea (MSR/MSPA) em leucena com e sem inoculação, com estirpe de rizóbio (NFB494, BR 814, BR 112 e IPA 209), Glomus etunicatum e Biofertilizante PK ${ }^{11}$.

\begin{tabular}{|c|c|c|c|c|}
\hline \multirow{2}{*}{ Tratamentos } & MSR & MSN & $\mathbf{T N}$ & MSR MSPA $^{-1}$ \\
\hline & $\left(\right.$ g vaso $\left.^{-1}\right)$ & $\left(\right.$ g vaso $\left.^{-1}\right)$ & $\left(\mathrm{mg} \mathrm{g}^{-1} \operatorname{vaso}^{-1}\right)$ & $\left(\mathrm{mg} \mathrm{N} \operatorname{vaso}^{-1}\right)$ \\
\hline NFB 494 -M-B & $8,6 \mathrm{~b}$ & 0,8 abcd & $31,7 \mathrm{a}$ & $0,5 \mathrm{a}$ \\
\hline NFB $494-M+B$ & $8,8 \mathrm{ab}$ & $0,9 a b$ & $31,7 \mathrm{a}$ & $0,5 \mathrm{a}$ \\
\hline NFB $494+\mathrm{M}+\mathrm{B}$ & $8,6 \mathrm{ab}$ & $0,9 \mathrm{a}$ & $33,1 \mathrm{a}$ & $0,5 \mathrm{a}$ \\
\hline NFB $494+\mathrm{M}-\mathrm{B}$ & $7,5 \mathrm{~b}$ & 0,8 abcd & $32,9 \mathrm{a}$ & $0,5 \mathrm{a}$ \\
\hline BR $814-M-B$ & $7,9 \mathrm{~b}$ & $0,6 \mathrm{bcd}$ & $31,3 \mathrm{a}$ & $0,4 \mathrm{a}$ \\
\hline BR $814-M+B$ & $8,3 \mathrm{~b}$ & 0,6 abcd & $31,1 \mathrm{a}$ & $0,4 \mathrm{a}$ \\
\hline BR $814+\mathrm{M}+\mathrm{B}$ & $8,5 \mathrm{~b}$ & $0,5 \mathrm{~d}$ & $31,7 \mathrm{a}$ & $0,4 \mathrm{a}$ \\
\hline BR $814+\mathrm{M}-\mathrm{B}$ & $8,8 \mathrm{ab}$ & $0,6 \mathrm{~cd}$ & $31,5 \mathrm{a}$ & $0,4 \mathrm{a}$ \\
\hline BR $112-M-B$ & $8,0 \mathrm{~b}$ & 0,9 abcd & $32,4 \mathrm{a}$ & $0,5 \mathrm{a}$ \\
\hline BR 112 -M+B & $8,7 \mathrm{ab}$ & 0,7 abcd & $30,6 \mathrm{a}$ & $0,5 \mathrm{a}$ \\
\hline BR $112+\mathrm{M}+\mathrm{B}$ & $7,8 \mathrm{~b}$ & 0,8 abcd & $33,2 \mathrm{a}$ & $0,4 \mathrm{a}$ \\
\hline BR $112+\mathrm{M}-\mathrm{B}$ & $7,7 \mathrm{~b}$ & 0,9 abcd & $32,9 \mathrm{a}$ & $0,5 \mathrm{a}$ \\
\hline IPA 209 -M-B & $8,4 \mathrm{~b}$ & 0,8 abcd & $32,5 \mathrm{a}$ & $0,4 \mathrm{a}$ \\
\hline IPA $209-\mathrm{M}+\mathrm{B}$ & $8,3 \mathrm{~b}$ & 0,8 abcd & $32,0 \mathrm{a}$ & $0,4 \mathrm{a}$ \\
\hline IPA $209+\mathrm{M}+\mathrm{B}$ & $8,3 \mathrm{~b}$ & 0,8 abcd & $32,4 \mathrm{a}$ & $0,4 \mathrm{a}$ \\
\hline IPA $209+$ +M-B & $8,8 \mathrm{ab}$ & 0,9 abcd & $32,5 \mathrm{a}$ & $0,5 \mathrm{a}$ \\
\hline$-\mathrm{R}-\mathrm{M}-\mathrm{B}$ & $8,8 \mathrm{ab}$ & $0,9 \mathrm{abc}$ & $32,9 \mathrm{a}$ & $0,6 \mathrm{a}$ \\
\hline$-\mathrm{R}-\mathrm{M}+\mathrm{B}$ & $8,4 \mathrm{~b}$ & $0,9 a b$ & $30,4 \mathrm{a}$ & $0,5 \mathrm{a}$ \\
\hline$-\mathrm{R}+\mathrm{M}+\mathrm{B}$ & $8,3 \mathrm{~b}$ & 0,8 abcd & $32,5 \mathrm{a}$ & $0,5 \mathrm{a}$ \\
\hline$-\mathrm{R}+\mathrm{M}-\mathrm{B}$ & $7,7 \mathrm{~b}$ & 0,7 abcd & $32,3 \mathrm{a}$ & $0,5 \mathrm{a}$ \\
\hline$+\mathrm{R}+\mathrm{PK}$ & $8,2 \mathrm{~b}$ & $0,6 \mathrm{~cd}$ & $30,3 \mathrm{a}$ & $0,4 \mathrm{a}$ \\
\hline$-\mathrm{R}+\mathrm{NPK}$ & $10,5 \mathrm{a}$ & 0,6 abcd & $28,5 \mathrm{a}$ & $0,5 \mathrm{a}$ \\
\hline $\mathrm{CV}(\%)$ & 8,59 & 17,68 & 8,24 & 16,60 \\
\hline
\end{tabular}

${ }^{(1)}$ M: fungo micorrízico arbuscular (Glomus etunicatum); B: biofertilizante de rocha fosfatada e potássica (PK), com enxofre inoculado com Acidithiobacillus; R: estirpe de rizóbio padrão; PK: adubo mineral - fósforo e potássio; NPK: adubo mineral - nitrogênio, fósforo e potássio; +: presente; -: ausente. Na coluna, as médias (3 repetições) seguidas da mesma letra não diferem estatisticamente entre si, ao nível de 5\% de probabilidade, pelo teste de Tukey. 
A variável relação (MSR/MSPA) também não apresentou diferença significativa $(\mathrm{p}<0,05)$, embora tenham sido superiores às plantas sem inoculação de rizóbio, Glomus etunicatum e Acidithiobacillus (-R-M-B). Em sequência, houve melhor resultado no tratamento com a estirpe de rizóbio (BR 112), na ausência do Glomus etunicatum e associada ao biofertilizante PK.

\section{Conclusões}

As plantas de leucena inoculadas com a estirpe de rizóbio $($ BR 814 $)+$ G. etunicatum + Biofertilizante PK apresentaram-se superiores na altura da planta, matéria seca da parte aérea (MSPA) e nitrogênio acumulado ( $\left.\mathrm{N}_{\mathrm{ac}}\right)$, confirmando a eficiência da inoculação conjunta.

As plantas de leucena apresentaram maior matéria seca de nódulos quando inoculadas com a NFB $494+G$. etunicatum + Biofertilizante PK com enxofre e Acidithiobacillus.

Na matéria seca da raiz, as plantas que receberam adubo mineral (NPK) e ausência de inoculação com rizóbio (-R+NPK) apresentaram-se superiores em relação aos demais tratamentos.

A inoculação com rizóbios efetivos em conjunto com o FMA- Glomus etunicatum e biofertilizante PK com enxofre e Acidithiobacillus torna-se uma alternativa para a adubação mineral, já que podem vir a substituir em parte essa adubação química na leucena.

\section{Agradecimentos}

À Fundação de Amparo à Ciência e Tecnologia do Estado de Pernambuco-Facepe pelo apoio financeiro. Ao Instituto Agronômico de Pernambuco-IPA e ao Laboratório de Biotecnologia - Núcleo de Fixação Biológica dos Trópicos da Universidade Federal Rural de Pernambuco-UFRPE pelo suporte técnico.

\section{Referências}

ANDRADE, M. M. M. et al. Fertilização mineral e biofertilizante de rochas com Bradyrhizobium e fungos micorrízicos arbusculares em caupi. Revista Brasileira de Ciências Agrárias, v. 4, n. 3, p. 289-292, jul./set. 2009. http://dx.doi.org/10.5039/agraria.v4i3a10

BREMNER, J. M. Total nitrogen. In: BLACK, C. A. (Ed.). Methods of soil analysis chemical and microbiological properties. Madison: American Society of Agronomy, 1965. parte 2, p. 1149-1178.

CAVALCANTI, F. J. A. et al. Recomendações de adubação para o estado de Pernambuco: $2^{\text {a }}$ aproximação. 3. ed. revisada. Recife: Instituto Agronômico de Pernambuco-IPA, 2008. 212 p.

CARVALHO, T. S.; MOREIRA, F. M. S. simbioses tripartites leguminosas - fungos micorrízicos e bactérias fixadoras de nitrogênio nodulíferas. In: SIQUEIRA, J. O. et al. (Eds.). Micorrizas no Brasil: os primeiros 30 anos. Lavras: Editora da UFLA, 2010. $716 \mathrm{p}$.

DRUMOND, M. A.; RIBASKI, J. Leucena (Leucaena leucocephala): leguminosa de uso múltiplo para o semiárido brasileiro. Petrolina: Embrapa Semiárido, 2010. 8 p. (Embrapa Semiárido. Comunicado Técnico, n. 142).
HOAGLAND, D.; ARNON, D.I. The water culture method for growing plants without soil. California: Agriculture Experimental Station, 1950. 32 p. (Agriculture Experiental Station. Circular, n. 347).

HUNGRIA, M.; ARAÚJO, R. S. Manual de métodos empregados em estudos de microbiologia agrícola. Brasília: Embrapa, 1994. 542 p.

JESUS, E. C.; SCHIAVO, J. A.; FARIA, S. M. Dependência de micorrizas para a nodulação de leguminosas arbóreas tropicais. Revista Árvore, v. 29, n. 4, p. 545-552, 2005. http://dx.doi. org/10.1590/S0100-67622005000400006

LIMA, R. C. M. et al. Eficiência e efeito residual de biofertilizantes de rochas com PK e enxofre com Acidithiobacillus em alface. Horticultura Brasileira, v. 25, p. 402-407, 2007. http://dx.doi. org/10.1590/S0102-05362007000300016

LIMA, P. C. F. Leucena. In: KIILL, L. H. P.; MENEZES, E. A. Espécies vegetais exóticas com potencialidades para o semiárido brasileiro. Brasília: Embrapa Informação Tecnológica, 2005. p. 157-205.

LIMA FILHO, J. M. P.; DRUMOND, M. A.; MACEDO, D. S. Comportamento fisiológico da Leucena e Albizio sob condições semiáridas. Pesquisa Agropecuária Brasileira, v. 27, n. 4, p. 537-542, 1992.

LORENZI, H. et al. Árvores exóticas no Brasil: madeireiras, ornamentais e aromáticas. Nova Odessa: Instituto Plantarum de Estudos da Flora, 2003. 368 p.

MOREIRA, F. M. S. et al. Bactérias fixadoras de $\mathrm{N}_{2}$ e fungos micorrízicos arbusculares em espécies florestais: avanços e aplicações biotecnológicas. parte 4 - microrganismos promotores do crescimento de plantas. In: FIGUEIREDO, M. V. B. et al. Biotecnologia aplicada à agricultura: textos de apoio e protocolos experimentais. Brasília: Embrapa, 2010. p. 439-477.

MOURA, P. M. et al. Eficiência de biofertilizantes de rochas com Acidithiobacillus em melão, n. Vale do São Francisco. Revista Brasileira de Ciências Agrárias, v. 2, n. 1, p. 1-7, jan./mar. 2007.

REIS, V. M. et al. Diazotróficos associativos e de vida livre: avanços e aplicações biotecnológicas. parte 4 - microrganismos promotores do crescimento de plantas. In: FIGUEIREDO, M. V. B. et al. Biotecnologia aplicada à agricultura: textos de apoio e protocolos experimentais. Brasília: Embrapa, 2010. p. 415-437.

SANCHEZ, P. A. Soil fertily and hunger in Africa. Science, v. 295, p. 2019-2020, 2002.

SANTOS, C. E. R. S. et al. Fixação simbiótica de $\mathrm{N}_{2}$ em leguminosas tropicais. parte I - fixação biológica do $\mathrm{N}_{2}$. In: FIGUEIREDO, M. V. B. et al. Microrganismos e agrobiodiversidade: o novo desafio para agricultura. Guaíba: Agrolivros, 2008. p. 17-41.

SILVA, M. O. et al. Diferentes fontes de P no desenvolvimento do meloeiro e disponibilidade de fósforo no solo. Revista Ciência Agronômica, v. 42, n. 2, p. 268-277, abr./jun. 2011. http://dx.doi. org/10.1590/S1806-66902011000200003

SOUZA, V. C. et al. Estudos sobre fungos micorrízicos. Revista Brasileira de Engenharia Agrícola e Ambiental, v. 10, n. 3, p. 612-618, 2006. http://dx.doi.org/10.1590/S141543662006000300011

STAMFORD, N. P. et al. Rock biofertilizers with Acidithiobacillus on sugarcane yield and nutrient uptake in Brazilian soil. Geomicrobiology, v. 23, p. 251-257, 2006. http://dx.doi. org/10.1080/01490450600760658

STAMFORD, N. P. et al. Effects of rock phosphate, sulphur with and without Acidithiobacillus and organic byproducts on mimosa (Mimosa caesalpiniifolia) grown in a Brazilian tableland soil. Tropical Grasslands, v. 39, p. 54- 61, 2005. 
STAMFORD, N. P. et al. Biofertilizantes de rocha fosfatada com Acidithiobacillus como adubação alternativa de caupi em solo com baixo P disponível. Revista Analytica, n. 9, p. 50, fev./mar. 2004.

STAMFORD, N. P.; CARVALHO, F. G. Fixação do $\mathrm{N}_{2}$ em leucena (Leucaena leucocephala) em solo da região semiárida brasileira submetido à salinização. Revista Brasileira de Ciência do Solo v. 23, p. 237-243, 1999.
TELES, M. M. et al. Quebra de dormência em sementes de leucena (Leucaena leucocephala (Lam.) de Wit). Revista Brasileira de Zootecnia, v. 29, n. 2, p. 387-391, mar./abr. 2000. http://dx.doi. org/10.1590/S1516-35982000000200010

VINCENT, J. M. A manual for the practical study of the root-nodule bacteria. London: International Biological Programme, 1970. 164 p. (IBP. Handbook, n. 15). 